\title{
Concerns of addiction to anaesthesiologists in the perioperative period
}

Volume 2 Issue 2 - 2015

\section{Introduction}

Substance abuse is increasing world-wide and has spread its evil tentacles in every continent. Addiction can be to licit/legal agents (eg. tobacco, alcohol) or to illicit/illegal drugs (heroin, cocaine, marijuana etc). Anesthesiologists, as perioperative physicians, have a vital role to play in the comprehensive care of such patients. ${ }^{1}$ It is equally important to realize the tremendous impact of drug addiction in the perioperative period. Creating awareness about the ill-effects of addiction in day-to-day life in general and in the peri-operative period in particular, is of paramount importance. Anesthesiologists need to be proactive in eliciting history of current or past drug abuse and in tackling this double-edged sword.

\section{Impact of addiction}

Drug abuse has a huge impact in the perioperative care of any addicted patient. Not only does it translate into increased costs, but also several medico-legal and socio-cultural problems. Bringing out a positive history of addiction or drug abuse can be a challenging task and urinary drug testing (UDS) has its own limitations. The complications following intravenous drug abuse are mainly three-fold: effects due to drug-toxicity; effects related to administration route; and effects arising out of social implications of drug-dependence. ${ }^{2}$ The anesthesiologists could take the responsibility for initiation of quitting the habit by creating awareness about its ill-effects on various body systems, including infections (cellulitis, endocarditis, abscesses and viremia), aneurysms and non-healing ulcers. They can highlight about the higher propensity for peri-operative complications. Pre-anesthetic visit must be elaborate, both in terms of time and assessment. Most of these patients would need intensive monitoring peri operatively. Many of them would require greater than normal doses of anesthetic and analgesic agents. The key to success lies in creating a fine balance, by avoiding both under-and over dosage. Ensuring adequate post-operative analgesia is of paramount importance, to prevent relapse to addiction by insufficient analgesia. ${ }^{3}$ Anesthesiologists must also be prepared to deal with withdrawal and abstinence syndrome. Greater care needs to taken in cases of combination or multiple drug addiction. To cement the impact of peri-operative counseling against drug addiction, long term follow-up advice and social support systems must be provided to these patients.

\section{Drugs commonly abused}

Anesthesiologists need to be aware of the common drugs which are being abused and their effects. They include (apart from tobacco and alcohol): heroin, ecstasy/MDMA, phencyclidine, cocaine, pentazocine, temazepam/diazepam, magic mushrooms, cannabis and solvents. ${ }^{4}$ There is a recent trend towards abuse of ketamine and propofol among medical professionals. Heroin, marijuana and cocaine are the most common (either alone or in combination). Heroin $^{5}$ (diacetylmorphine or diamorphine) can be abused in several ways: sniffing (snorting); smoking (chasing the dragon); subcutaneous injection (skin popping); intravenous injection (mainlining???); oral

\author{
Rakesh Garg,' Uma R Hariharan² \\ 'Department of Anaesthesiology, India \\ ${ }^{2}$ Department of Anaesthesia and Intensive Care, India
}

Correspondence: Rakesh Garg, Department of Anaesthesiology, Pain and Palliative Care, Dr BRAIRCH,All India Institute of Medical Sciences, Room No. 139, Ist floor, Ansari Nagar, New Delhi- I I0029, India, Tel +9l 9810394950, +9l 9868398335,Email drrgarg@hotmail.com

Received: March 20, 2015 | Published: April 09, 2015

intake; or in combination with cocaine (speed ball). It is metabolized to morphine, excreted in urine as free and un conjugated morphine. Overdose can lead to coma, rhabdomyolysis, hypotension, aspiration pneumonia, pulmonary edema, hypoxia, acidosis and dehydration. There is a high rate of viral and bacterial infection, leading to glomerulonephritis, local pyogenic abscess, endocarditis, secondary amyloidosis, HAN (Heroin associated nephropathy) and HIVAN (HIVassociated nephropathy from Street Heroin). Cocaine ${ }^{6}$ is an alkaloid extracted from erythroxylon coca. It is absorbed through the mucous membrane and smoked or injected. Eighty to $90 \%$ is metabolized, rest is excreted unchanged. Euphoria is caused by blocking of dopamine reuptake and hypertensive effects are due to inhibition of reuptake of noradrenaline. It has local anesthetic action. Acute renal failure from rhabdomyolysis and renal infarction is also a possibility. Muscle ischemia, contamination of cocaine with heavy metals, crack lung and syndrome of cocaine-induced premature coronary artery disease are serious side-effects. Ecstasy or MDMA (3,4-methylene dioxy methamphet amine) undergoes liver metabolism and renal excretion. ${ }^{7}$ Oral intake in group dancing (rave parties or clubs) leads to hyperthermia, fever, dehydration, convulsions, DIC (disseminated intravascular coagulation), rhabdomyolysis and acute renal failure (ARF). If they drink large quantities of water to prevent dehydration, then they may develop dilutional hyponatremia (due to SIADH). Sympathomimetic effects can cause accelerated hypertension, urinary retention or arrhythmias.

Marijuana or cannabis (street names include ganja/reefer/dope/ grass/weed/bhang) overdose (common in accidental/intentional ingestion of drug mixed with food or smoking joints) causes tachycardia, dry mouth, dilated pupils, arrhythmias, memory loss, impaired motor skills increasing propensity for fatal accidents, acute toxic psychosis, panic attacks or loss of consciousness. ${ }^{8}$ It is the most abused drug among the youth in United States and its "high" is produced by the active compound called THC (delta-9-tetra hydro cannabinol). Benzodiazepines are commonly abused as they are 
easily available over-the-counter drugs or by thefts. ${ }^{9}$ Inadvertent intra-arterial injection can cause acute renal failure or limb ischemia. Panaeolus muscaria and Psilocybe are hallucinogens (liberty cap or magic mushrooms). ${ }^{10}$ Inadvertent ingestion of poisonous mushroom species can cause nephrotoxicity and oliguric renal failure. Volatile substances like toluene and benzene can be abused (glue sniffing) as they produce short term hallucinations. Solvent abuse can cause serious complications affecting kidney, lung and the cardiovascular system.

\section{Pre-anesthetic evaluation}

Drug abuse patients can present to the anesthesiologists for a variety of reasons, either emergency or elective or during labor or for trauma care. Most of the illicit drugs impair the motor skills and reaction time and are responsible for serious accidents, causing death or disability due to trauma. In elective cases, data about drug abuse may be obtained at the time of preadmission testing or in preoperative area (subjective), or during urine and blood testing (objective). There are several constraints of UDS, ${ }^{11}$ as many standard tests report only as positive for opiates and fentanyl cannot be detected. Only current or recent abuse can be detected and not past abuse. Immunoassays for specific opioids can have false-positive results and they must be confirmed by gas chromatography, which is both time-consuming and expensive. The modified CAGE-AID (Conjoint screening questionnaires for alcohol and other drug abuse) questionnaire can be utilized to elicit drug or alcohol abuse. ${ }^{12}$ Detailed questioning must also be done in a "clean addict", who was previously an opioid addict and successfully undergone withdrawal therapy and currently not on any pharmacotherapy. Some patients may present to the pre anaesthetic clinic who may be on opioid de-addiction therapy. Oral methadone is the commonest drug used for this purpose. ${ }^{13}$ It is a long-acting opioid agonist with some activity on NMBA (N-methyl-D-aspartate) receptors and given in a dose of 30-40 $\mathrm{mg}$ /day to suppress withdrawal symptoms. It is advisable to continue methadone maintenance therapy till the morning of surgery. Buprenorphine (1000 times higher affinity for $\mathrm{mu}$ receptors) is a partial opioid agonist, which relieves drug cravings without producing dangerous side effects. ${ }^{14}$ Its advantages are that, it has a ceiling effect and a long half-life. Suboxone is a combination of buprenorphine and naloxone given intravenously, with increased therapeutic window and relative ease of use, as daily supervision is not needed.

Some opioid dependant patients may be on an extended release preparation of opioid antagonist, Naltrexone, given as monthly injections. It is imperative to determine the time of last dose of opioids to finalize our choice of drugs. ${ }^{15}$ Naltrexone should be discontinued at least 24-72 hours prior, if opioid based anesthesia is planned and then restarted after 5-7days, remembering to cover the intervening period with non-opioid analgesics. If these agents are continued, then it is better to avoid intra-operative opioids to prevent precipitation of withdrawal or other adverse reactions. Some patients may require an early psychiatric consult for malingering and anxiety states or depression. Preoperative assessment must also focus on detecting viral markers suggestive of HBV (hepatitis B), HCV (Hepatitis C) or HIV (Human immunodeficiency virus) infection, especially in chronic parenteral drug abusers. Needless to say, universal precautions and complete asepsis should be practiced by all health care workers in handling such patients. ${ }^{16}$

\section{Intra-operative management}

The three prime aspects of intra-operative management include managing intoxication, preventing or treating withdrawal, and achieving effective analgesia. ${ }^{17}$ Stress relief is of colossal importance, especially by preventing under-dosing. Anesthetic agents must be titrated to individual patient needs and greater than normal doses are usually required. Moreover, these patients may also have difficult venous access and ultrasound guidance can be particularly beneficial in this scenario. ${ }^{18}$ There may be nutritional deficiencies due to chronic malnutrition, which can lead to hypoproteinemia and increased free fraction of protein bound drugs. Hypotension on induction is common due to concurrent dehydration or hypovolemia. Pre-existing electrolyte imbalances must be corrected. In opioid addicts, a nonopioid based anesthesia can be safely administered. This regime includes ketamine, benzodiazepines, volatile anesthetics, paracetamol NSAIDs and COX-2 inhibitors. Regional techniques are a boon in such patients for both anesthesia and analgesia. Ultrasound-guided nerve or plexus blocks can be performed pre-emptively for decreasing anesthetic requirements and extended for post-operative pain relief. Electronic patient controlled analgesia or PCA (intravenous, epidural or continuous plexus blocks) pumps go a long way in gaining the confidence of the patient and giving him a feeling of self-control. ${ }^{19}$ If an opioid- based regime is chosen, then it must be remembered that the opioid dose requirement is increased by $50-100 \% .{ }^{20}$ Opioid therapy in a patient presenting after prolonged abstinence or successful opioid withdrawal can cause an exaggerated response and lead to delayed recovery from anesthesia or prolonged respiratory depression requiring post-operative ventilatory support. At the same time, inadequate analgesic dosing can re-activate addiction in abstinent patients. Remifentanil infusion can be beneficial in view of its short duration of action and better safety profile. Prevention of craving is of over-riding importance..$^{21}$ Treatment of withdrawal syndrome can be accomplished with clonidine, tramadol and loperamide. ${ }^{22}$ It is better to avoid mixed opioid agonist-antagonists like nalbuphine, butorphanol and pentazocine in patients on opioid withdrawal regime. A drugabusing parturient can be quite challenging at any stage of presentation: antenatal emergencies, labor analgesia, caesarian section or postpartum intensive care. Early anesthetic consultation during antenatal visits is recommended. ${ }^{23}$ Labor epidural with local anesthetics can be given, which can be continued for operative delivery if required. If general anesthesia is warranted for emergency section, then a nonopioid- based anesthesia with full stomach precautions and with preparations for neonatal resuscitation can be administered. These women are prone for post-operative complications like PPH (postpartum hemorrhage), infection, embolism and post-partum blues.

\section{Post-operative care}

The main goals in the postoperative period include ensuring both patient comfort and safety. ${ }^{24}$ Alternate methods of pain relief must be practiced with co-analgesics like intravenous paracetamol, liposomal bupivacaine, $\alpha 2$ agonists (clonidine and dexmedetomidine), COX2 inhibitors, NSAID`s, pregabalin and gabapentin. Nerve blocks and PCA must be continued into the postoperative period for a smooth recovery. Apart from infection control and universal precautions, DVT (deep vein thrombosis) and PONV (postoperative nausea vomiting) prophylaxis must be instituted. If patient was previously on buprenorphine therapy, then there can be four pathways for acute pain management: continue maintenance therapy, patients will require higher than usual doses of short-acting opioid to achieve desired effect due to persistence of buprenorphine on receptors; divide buprenorphine into TDS (three times a day) dosing in less invasive surgeries due its inherent analgesic properties; discontinue buprenorphine and give full opioid analgesics and be watchful of withdrawal; or convert to methadone $30-40 \mathrm{mg} /$ day in standard TDS dosing and documentation of methadone therapy with the authorized de-addiction centres. ${ }^{25}$ 


\section{Conclusion}

The impact of addiction in the perioperative period is multi-faceted and dynamic. Awareness about the growing problem of drug abuse and its evils must be created both among the perioperative physicians and the patients. Lack of standard protocols in anesthetic management of addicted patients and the unpredictable nature of drug interactions is responsible for precipitation of withdrawal or occurrence of complications. Drug abuse can affect almost all body systems. American Society of Anesthesiologists (ASA) Guidelines on smoking cessation ("AAR" principle of ask, advice and refer) must be followed for tobacco addicts in the preanesthetic clinic. ${ }^{26}$ Anesthesiologist can act as pioneers in counseling for deaddiction??. Nevertheless, adequate analgesia must be provided and a fine balance of optimum anesthesia created by avoiding both under-and over-dosage. Addicted patients must be dealt with compassion, pharmacotherapy for deaddiction must not be first initiated in the perioperative period and adequate analgesia must never be withheld. It is high time to start specialized training of anesthesiologists in dealing with drug addicted patients for a successful perioperative outcome.

\section{Acknowledgments}

None.

\section{Funding details}

None.

\section{Conflicts of interest}

Authors declare that there is no conflict of interest.

\section{References}

1. Steadman JL, Birnbach DJ. Patients on party drugs undergoing anesthesia. Curr Opin Anaesthesiol. 2003;16(2):147-152.

2. Lucas CE. The impact of street drugs on trauma care. J Trauma. 2005;59(3 Suppl):S57-S60.

3. Jage J, Heid F. Anesthesia and analgesia in addicts:basis for establishing a standard operating procedure. Anaesthesist. 2006;55(6):611-628.

4. Crowe AV, Howse M, Bell GM, et al. Substance abuse and the kidney. QJM. 2000;93(3):147-152.

5. Hosztafi S. Heroin, part III:the pharmacology of heroin. Acta Pharm Hung. 2000;73(7):197-205.

6. Benowitz NL. Clinical pharmacology and toxicology of cocaine. Pharmacol Toxicol. 1993;72(1):3-12.

7. Verebey K, Alrazi J, Jaffe JH. The complications of [Math Processing Error] (MDMA). JAMA. 1998;259(11):1649-1650.

8. Iyalomhe GB. Cannabis abuse and addiction:a contemporary literature review. Niger J Med. 2009;18(2):128-133.
9. Blair SD, Holcombe C, Coombes EN, etal. Leg ischemia secondary to nonmedical injection of temazepam. Lancet. 1991;338(8779):1393-1394.

10. Raff E, Halloran F, Kjellstrand CM. Renal failure after eating [Math Processing Error] mushrooms. Can Med Assoc J. 1992;147(9):1339-1341.

11. Moeller K, Lee K, Kissack J. Urine drug screening:practical guide for clinicians. Mayo Clin Proc. 2008;83(1):66-76.

12. Brown RL, Rounds LA. Conjoint screening questionnaires for alcohol and other drug abuse:criterion validity in a primary care practice. Wis Med J. 1995;94(3):135-140.

13. Kleber HD. Pharmacologic treatments for heroin and cocaine dependence. Amm J Addict. 2003;12(2):S5-S18.

14. Ling W. Buprenorphine for opioid dependence. Expert Rev Neurother. 2009;9(5):609-616.

15. Dunbar JL, Turncliff RZ, Dong Q, et al. Single- and multiple-dose pharmacokinetics of long-acting injectable naltrexone. Alcohol Clin Exp Res. 2006;30(3):480-490.

16. Ben Diane MK, Feroni I, Poncet M, et al. Chief health risks associated with intravenous heroin and cocaine abuse. Presse Med. 2000;29(8):453-457.

17. Vadivelu N, Mitra S, Kaye AD, et al. Perioperative analgesia and challenges in the drug-addicted and drug-dependent patient. Best Pract Res Clin Anaesthesiol. 2004;28(1):91-101.

18. Constantino TG, Parikh AK, Satz WA, et al. Ultrasonography-guided peripheral intravenous access versus traditional approaches in patients with difficult intravenous access. Ann Emerg Med. 2005;46(5):456-461.

19. Lerchl-Wanie G, Angster R. Perioperative analgesia for opioid tolerant patients. Anaesthesist. 2010;59(7):657-670.

20. Mitra S, Sinatra RS. Perioperative management of acute pain in the opioid-dependent patient. Anesthesiology. 2004;101(1):212-227.

21. Goyal R, Khurana G, Jindal P, et al. Anesthesia for opioid addict:Challenges for perioperative physician. $J$ Anaesthesiol Clin Pharmacol. 2013;29(3):394-396.

22. Stromer W, Michaeli K, Sandner-Kiesling A. Perioperative pain therapy in opioid abuse. Eur J Anaesthesiol. 2013;30(2):55-64.

23. Kuczkowski KM. Anesthetic implications of drug abuse in pregnancy. $J$ Clin Anesth. 2005;15(5):382-394.

24. Vaghari B, Baratta JL, Gandhi K. Perioperative Approach to Patients with Opioid Abuse and Tolerance. Anesthesiology News. 2013.

25. Alford DP, Compton P, Samet JH. Acute pain management for patients receiving maintenance methadone or buprenorphine therapy. Ann Intern Med. 2006;144(2):127-134.

26. Warner DO. Feasibility of tobacco interventions in anesthesiology practices:A pilot study. Anesthesiology. 2009;110(6):1223-1228. 